\title{
ATIVIDADES EDUCATIVAS ESTIMULANDO O AUTOCUIDADO E PREVENÇÃO DO CÂNCER FEMININO
}

\section{EDUCATIONAL ACTIVITIES IN ENCOURAGING SELF-CARE AND PREVENTION OF FEMALE CANCER}

\section{ACTIVIDADES EDUCATIVAS ESTIMULANDO EL AUTOCUIDADO Y PREVENCIÓN DEL CÁNCER FEMENINO}

\author{
Lorena Campos Mendes ${ }^{1}$, Thaís Cristina Elias ${ }^{2}$, Taciana Nunes dos Santos ${ }^{3}$, Erika Mendes \\ Tayar $^{4}$, Sueli da Silva Riul ${ }^{5}$
}

\begin{abstract}
RESUMO
Objetivo: descrever as práticas educativas, realizadas com estudantes do ensino médio noturno de escolas estaduais do município de Uberaba/MG, a respeito de ações de incentivo ao autocuidado em relação à prevenção e diagnóstico do câncer de mama e do colo do útero. Método: trata-se de um relato de experiência desenvolvido com alunas de escolas estaduais de caráter regular e modalidade presencial do período noturno. Resultados: foi beneficiado um grupo de aproximadamente 1040 estudantes. Notou-se que, de um modo geral, as mulheres eram carentes de informações relacionadas à temática e que possuíam um déficit de conhecimento maior referente à Autopalpação das Mamas em relação ao exame Papanicolaou. Considerações finais: observou-se que a transmissão de informações neste contexto é de extrema importância, e apresenta-se como uma ferramenta fundamental na assistência de Enfermagem.
\end{abstract}

Descritores: Autoexame de mama; Educação em saúde; Enfermagem; Esfregaço vaginal; Saúde da mulher.

\begin{abstract}
Aim: this study aimed to describe educational practices conducted with students of night schools state, in the city of Uberaba/MG, about actions to encourage self-care in relation to the prevention and diagnosis of breast cancer and cervical cancer. Method: this is an experience report developed with students from state schools on a regular basis and face modality of nighttime. Results: it benefited a group of approximately 1040 students. It was noted that, in general, women were deprived of information related to the subject and who had greater knowledge deficit regarding the breast self-examination regarding the vaginal smears. Final considerations: it was observed that the transmission of information in this
\end{abstract}

1 Enfermeira. Mestre em Atenção à Saúde. Universidade Federal do Triângulo Mineiro (UFTM). E-mail: lorena_camposmendes@hotmail.com

2 Enfermeira. Mestre em Atenção à Saúde. Universidade Federal do Triângulo Mineiro (UFTM). E-mail: thaiscelias2@hotmail.com

3 Acadêmica do Centro de Graduação em Enfermagem da Universidade Federal do Triângulo Mineiro (UFTM).

E-mail: taciana.nunes@hotmail.com

4 Acadêmica do Centro de Graduação em Enfermagem da Universidade Federal do Triângulo Mineiro (UFTM).

E-mail: erikatayar@hotmail.com

5 Enfermeira e obstetríz. Mestre e doutora em Enfermagem Fundamental. Professora Associada do Programa de Pós Graduação em Atenção à Saúde da Universidade Federal do Triângulo Mineiro (UFTM). E-mail: sueliriul@terra.com.br 
context is extremely important, and presents itself as an essential tool in nursing care.

Descriptors: Breast self-examination; Health education; Nursing; Vaginal smears; Women's health.

\section{RESUMEN}

Objetivo: describir las prácticas educativas, realizadas con estudiantes de la enseñanza media nocturna de escuelas estaduales del municipio de Uberaba/MG, a respecto de acciones de incentivo al auto cuidado en relación à prevención y diagnóstico del cáncer de mama y del cuello del útero. Método: se trata de un relato de experiencia desarrollado con alumnas de escuelas estaduales de carácter regular y modalidad presencial del período nocturno. Resultados: fue beneficiado un grupo de aproximadamente 1040 estudiantes. Se notó que, de una manera general, las mujeres eran carentes de informaciones relacionadas a la temática y que poseían un déficit de conocimientos mayor referente a la Auto palpación de las Mamas en relación al examen Papanicolaou. Consideraciones finales: se observó que la transmisión de informaciones en este contexto es de extrema importancia, y se presenta como una herramienta fundamental en la asistencia de Enfermería.

Descriptores: Autoexamen de mama; Educación en salud; Enfermería; Frotis Vaginal; Salud de la mujer.

\section{INTRODUÇÃO}

O Câncer (CA) atualmente é considerado um problema de saúde pública, uma vez que representa a segunda causa de morte por doença no mundo assim como no Brasil, sendo precedida por aquela causada pelas doenças cardiovasculares. ${ }^{1} \mathrm{O}$ câncer de mama é o tipo que mais acomete mulheres em todo o mundo, tanto em países em desenvolvimento quanto em países desenvolvidos. De acordo com dados do Instituto Nacional de Câncer (INCA), o CA de mama é a neoplasia mais comum no sexo feminino no Brasil, quando, para os anos de 2016-2017 serão esperados 57.960 casos novos com um risco estimado de 56,20 casos a cada 100 mil mulheres. ${ }^{2}$

Embora o CA de mama possa ser considerado de bom prognóstico quando detectado e tratado de maneira oportuna, as taxas de mortalidade no Brasil continuam elevadas, provavelmente porque a doença ainda é diagnosticada tardiamente em estágios já avançados. ${ }^{3}$

Já o CA do colo do útero, representa o quarto tipo de neoplasia maligna mais comum entre as mulheres no mundo e está diretamente vinculado ao grau de desenvolvimento do país, sendo duas vezes mais incidente em países menos desenvolvidos. O número de casos novos de CA cérvico uterino esperado para o Brasil nos anos de 2016-2017 será de 16.340, com um risco estimado de 15,85 casos a cada 100 mil mulheres. ${ }^{2}$

Com base nessas informações percebe-se a importância das atividades educativas na orientação para detecção do CA. A Autopalpação das Mamas (APM) e o exame Papanicolaou promovem o autocuidado e permitem que a mulher 
tenha maior intimidade e compreensão de si, proporcionando benefício a sua saúde e detecção dessas neoplasias.

Embora os exames preventivos para o CA de mama e do colo do útero sejam amplamente divulgados pela mídia e pelos profissionais de saúde, estudos mostram que seu conhecimento não é completo e homogêneo. ${ }^{4,5}$ A equipe de Enfermagem desempenha, neste contexto, uma importante função, tendo como princípio a implementação de práticas educativas que estimule e capacite o indivíduo, tornando-o sujeito ativo no processo saúde-doença, além de aumentar a frequência e adesão aos exames citados. ${ }^{6}$

Diante do exposto, e considerando que a APM e o Exame de Papanicolaou são exames simples, de baixo custo e que favorecem a detecção dessas patologias, justifica-se a necessidade da realização de atividades educativas na orientação para o autocuidado, tornando as mulheres sujeito ativo no processo saúde-doença, aumentando a frequência e adesão das mesmas aos exames citados.

Assim, o presente relato teve como objetivo descrever as práticas educativas, realizadas com estudantes do ensino médio noturno de escolas estaduais do município de Uberaba/MG, a respeito de ações de incentivo ao autocuidado em relação à prevenção e diagnóstico precoce do CA de mama e do colo do útero.

\section{MÉTODO}

Trata-se de um relato de experiência oriundo de um trabalho desenvolvido no curso de mestrado do Programa de Pós-Graduação em Atenção à Saúde (PPGAS), com a participação de acadêmicas do curso de graduação em Enfermagem da Universidade Federal do Triângulo Mineiro (UFTM), acadêmicas estas que se disponibilizaram a contribuir com a iniciativa, a título de atividade de extensão universitária. $\mathrm{O}$ referido trabalho foi desenvolvido com as mulheres matriculadas em escolas estaduais de caráter regular e modalidade presencial do período noturno do município de Uberaba/MG que estiveram presentes no momento da atividade.

As atividades foram realizadas no período de fevereiro a março de 2014, sendo incluídas todas as escolas estaduais que ofereciam o ensino médio noturno na zona urbana do município, somando um total de vinte instituições. As atividades educativas tiveram duração média de 45 minutos e foram desenvolvidas na forma de palestras, baseadas no diálogo e na troca de saberes. Em cada escola participante, as mulheres foram convidadas a formar um único grupo e a atividade foi desenvolvida uma vez em cada escola. Como recursos didáticos foram utilizados a explanação verbal dos temas APM e exame Papanicolaou, e recursos audiovisuais 
como banner e instrumentos de coleta do Papanicolaou.

No desenvolvimento da atividade procedeu-se inicialmente a uma explanação sumária dos temas em questão, com auxilio dos recursos didáticos elaborados para este fim, e em seguida era aberto espaço para participação das mulheres, que podiam então fazer suas colocações livremente, seja apresentando suas dúvidas ou observações, seja apresentando depoimentos ou esclarecimentos. Neste momento, pósgraduandas e acadêmicas se colocavam à disposição para responder às dúvidas, oferecer informações complementares e dar continuidade à argumentação das participantes.

O período noturno foi selecionado como momento oportuno para desenvolvimento da atividade, uma vez que neste período de ensino encontram-se mulheres que compõem a população-alvo das atividades preventivas dos cânceres de mama e colo uterino, a saber, mulheres entre 25 e 64 anos. $^{2}$

Para o desenvolvimento do presente trabalho, que faz parte de um estudo maior, foi solicitado o parecer e aprovação do Comitê de Ética em Pesquisa (CEP) da UFTM sendo aprovado sob o protocolo CEP/UFTM: 2585.
Foram beneficiadas com as atividades educativas um grupo de aproximadamente $1040 \quad$ estudantes. Observou-se que a mediana de idade das mulheres participantes foi de 24 anos, muitas exerciam atividade não remunerada, já que grande parte apenas estudava, e grande parte também era procedente do próprio município. A situação conjugal evidenciada durante a realização das palestras foi solteira. A recepção das 20 escolas para que a atividade fosse realizada foi fundamental, uma vez que muitos diretores julgaram a atividade importante e citaram problemas frequentes relacionados à saúde da mulher, como o desconhecimento frente aos métodos diagnósticos do CA de mama e do colo do útero, iniciação precoce da atividade sexual, uso inadequado de métodos contraceptivos e gravidez na adolescência.

Durante a realização do trabalho proposto, foi notória a carência de informações relacionadas à temática, além de outros assuntos correlatos como métodos contraceptivos e doenças sexualmente transmissíveis. Observou-se que, em um contexto geral, as mulheres possuíam um déficit de conhecimento maior referente à $\mathrm{APM}$ em relação ao exame Papanicolaou, fato evidenciado pelo volume de questionamentos levantados acerca do assunto.

\section{RESULTADOS}


Em relação à $\mathrm{APM}$ as principais dúvidas relacionaram-se à diferença entre nódulos benignos e malignos, idade para se iniciar a APM, diferença entre APM e mamografia, fatores de risco e proteção, e os principais sinais e sintomas do CA de mama. Percebeu-se que, grande parte das mulheres associava erroneamente APM com diagnóstico precoce.

Observou-se também que, embora as mulheres afirmassem conhecer a APM, pouco se sabia sobre a prática e o início de realização, além disso, muitas a confundiam como sinônimo da mamografia. No geral, notou-se que o tema CA de mama causava maior desconforto e medo nas participantes.

Referente ao Papanicolaou percebeu-se que muitas conheciam a finalidade e a periodicidade do exame, porém foi grande o número de mulheres que confundiam quando iniciar o exame, não procurando, assim, os serviços de saúde no momento ideal. Ainda que muitas já tivessem ouvido falar sobre o Papilomavírus Humano (HPV), desconheciam sua relação com o CA de colo de útero. Uma dúvida frequente entre as alunas se relacionou à imunização contra o HPV e sua efetividade.

De forma geral, diretores, professores e alunas demonstraram-se satisfeitos com as atividades. As mulheres manifestaram interesse nos assuntos apresentados, uma vez que participaram ativamente da atividade proposta.

\section{DISCUSSÃO}

A neoplasia mamária quando detectada em estágios iniciais apresenta prognóstico favorável, com o uso de terapias mais efetivas e menos agressivas. Para que isso se realize se faz necessária a implementação de estratégias para diagnóstico precoce do $\mathrm{CA}$ de mama através da educação da mulher para o reconhecimento dos sinais e sintomas característicos da doença, assim como o acesso facilitado aos serviços de saúde. ${ }^{7}$

Durante a realização das atividades educativas observou-se que, muitas mulheres associavam APM com diagnóstico precoce, o que é um erro. Corroborando com essa observação, um trabalho realizado em Uberaba/MG (2013), encontrou que grande parte das participantes do estudo apresentou desconhecimento com relação à finalidade do autoexame de mamas (AEM), sendo que $52,5 \%$ destas referiram que o AEM permite localizar nódulos precocemente e $31,5 \%$, ser este um exame preventivo contra o CA de mama. ${ }^{8}$

Dados de 2013 disponibilizados pelo INCA apontam que a maioria das mulheres com diagnóstico de CA de mama identifica-o por meio da palpação ocasional das mamas, e não da prática do 
AEM. Assim, atualmente, a estratégia utilizada é a 'breast awareness' que significa estar alerta para a saúde das mamas. ${ }^{7,9}$ Cabe ressaltar aqui a diferença entre AEM e APM, sendo o AEM uma estratégia propedêutica que inclui técnica e periodicidade definidas e a APM uma atitude consciente de atenção ao estado geral das mamas sem, contudo, observância de rigores técnicos para sua aplicação. ${ }^{7}$

Assim, a prática da APM ressalta a importância do diagnóstico precoce, de modo a orientar a população feminina sobre as mudanças normais das mamas em diferentes momentos do ciclo de vida e a divulgar os principais sinais e sintomas do CA, estimulando as mulheres a procurarem esclarecimento médico, a participarem de ações de rastreamento e a realizarem a autopalpação ocasional das mamas, sem método e período estabelecidos. ${ }^{7,9}$

Sob este prisma, salienta-se que tanto o AEM quanto a APM são exames simples e importantes no autoconhecimento e na ação de controle do CA de mama. Em consonância a esta afirmação, pesquisa realizada com usuárias de uma Unidade de Saúde da Família, identificou que aproximadamente 19,7\% das praticantes do AEM detectaram alterações mamárias ao realizar a técnica. ${ }^{10}$

Referente ao Papanicolaou, de acordo com as diretrizes brasileiras, deve ser realizado em todas as mulheres com vida sexual ativa, principalmente naquelas que estiverem na faixa etária de 25 a 64 anos de idade, definida como populaçãoalvo, a cada três anos, após dois exames anuais normais consecutivos. Ressalta-se que o CA de colo de útero apresenta alto potencial de cura quando diagnosticado precocemente, sendo que o exame Papanicolaou se destaca como a principal estratégia nos programas de rastreamento. $^{1,2}$

Com relação às barreiras encontradas para a realização do Papanicolaou, trabalho desenvolvido em São Paulo/SP (2008) apontou que, das 41 adolescentes que afirmaram nunca terem realizado o exame, $26,8 \%$ disseram que não o fizeram por medo e $19,5 \%$ por vergonha. ${ }^{11}$ Ressalta-se também o estudo realizado em Toledo/PR (2009) no qual os autores evidenciaram que o conhecimento das mulheres a respeito do Papanicolaou foi superficial e equivocado, sendo que muitas realizam o exame mais pelo aspecto curativo do que preventivo, ou seja, acreditando que a realização do exame, que é diagnóstico, tem potencial curativo. ${ }^{12}$

Dessa forma, enfatiza-se a necessidade de ampliação de informações, através de campanhas de educação em saúde, enfatizando a periodicidade de realização dos exames de Papanicolaou e APM, e de se repensar as metodologias de 
ensino utilizadas objetivando-se maior adesão aos exames e, consequentemente, redução da mortalidade por causas relacionadas. $^{11,13}$ Ressalta-se ainda a importância da efetivação e inserção de atividades de saúde no contexto escolar e social. $^{14}$

\section{CONSIDERAÇÕES FINAIS}

Reforça-se a importância da educação em saúde, para que as mulheres adquiram conhecimento e se tornem agentes de informação, visando à promoção da saúde e o estímulo ao autocuidado. Assim, aponta-se a necessidade da criação de estratégias e programas que favoreçam a redução do número de casos do agravo citado, despertando nas mulheres o interesse para a realização dos exames de rastreamento do CA de mama e de colo do útero. Considera-se que a atividade educativa gerou um impacto educacional positivo posto que a maioria das alunas demonstrou interesse na continuidade desta, buscando a troca de experiências e o ensino sobre questões relacionadas à temática.

A principal limitação deste trabalho consistiu o fato de que a atividade foi pontual e desenvolvida uma única vez em cada escola, fato que inviabiliza a exploração ampliada e aprofundada do tema. Contudo, há perspectiva de continuidade como proposta de programa de extensão universitária do Centro de Graduação em Enfermagem da UFTM, a ser desenvolvido em parceria com a Delegacia Estadual de Educação no município, iniciativa que contribuirá com a divulgação das recomendações do Ministério da Saúde e do INCA acerca do controle dos cânceres de colo do útero e mama, bem como oportunizará as acadêmicas de Enfermagem o desenvolvimento de habilidades na área da educação em saúde.

\section{REFERÊNCIAS}

1. Ministério da Saúde. Instituto Nacional de Câncer (BR). Ações de enfermagem para o controle do câncer: uma proposta de integração ensino-serviço. 3ed. Rio de Janeiro: INCA; 2008.

2. Ministério da Saúde. Instituto Nacional de Câncer José Alencar Gomes da Silva (BR). Estimativa 2016: incidência de câncer no Brasil. Rio de Janeiro: INCA; 2015.

3. Ministério da Saúde. Instituto Nacional de Câncer (BR). Estimativa 2012: incidência de câncer no Brasil. Rio de Janeiro: INCA; 2011.

4. Silva SR, Lício FC, Borges LV, Mendes LC, Vicente NG, Gomes NS. Atividades educativas na área da saúde da mulher: um relato de experiência. REAS. 2012;1(1):106-12.

5. Valente CA, Andrade V, Soares MBO, Silva SR. Women's knowledge about the papanicolaou exam. Rev. Esc. Enferm. USP. 2009;43(Esp2):1193-8.

6. Silva IJ, Oliveira FV, Silva SED, Polaro SHI, Radünz V, Santos EKA, et al. Care, self-care and caring for yourself: a paradigmatic understanding thought for nursing care. Rev. Esc. Enferm. USP. 2009;43(3):697-703. 
7. Ministério da Saúde (BR). Cadernos de atenção básica: controle dos cânceres do colo do útero e da mama. Brasília: MS; 2013.

8. Mendes LC, Silveira CF, Silva SR. Conhecimento de mulheres a respeito do exame de Papanicolaou e do Autoexame das Mamas. REAS. 2013; 2(3):4-17.

9. Thornton H, Pillarisetti RR. 'Breast awareness' and 'breast self-examination' are not the same. What do these terms mean? Why are they confused? What can we do? Eur J Cancer. 2008;44(15):211821.

10. Silva NRA, Soares DA, de Jesus SR. Conocimiento y práctica del autoexamen de mamas por usuarias de la Unidad de Salud de la Familia. Enferm. glob. 2013;29:463-76.

11. Cirino FMSB, Nichiata LYI, Borges AV. Conhecimento, atitudes e práticas na prevenção do câncer de colo uterino e HPV em adolescentes. Esc. Anna Nery. 2010;14(1):126-34.

12. Oliveira SL, Almeida ACH. A percepção das mulheres frente ao exame de
Papanicolaou: da observação ao entendimento. Cogitare enferm. 2009; 14(3):518-26.

13. Abril FGM, Díaz JMO, Fuentes NAV, Pacheco ALM, Amaya GH. Factores asociados a la práctica correcta del autoexamen de mama en mujeres de Tunja (Colombia). Invest Educ Enferm. 2012;30(1):18-27.

14. Molina MCC, Stoppiglia PGS, Martins CBG, Alencastro LCS. Conhecimento de adolescentes do ensino médio quanto aos métodos contraceptivos. O Mundo da Saúde. 2015;39(1):22-31.

AGRADECIMENTOS: ao Programa de Apoio a Planos de Reestruturação e Expansão das Universidades Federais - REUNI, pelo apoio financeiro na forma de bolsa de estudos, nível mestrado.

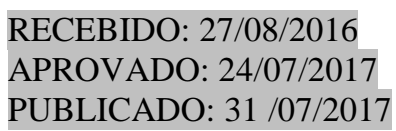

